\title{
Robotic Surgery in Children
}

\author{
HIROYUKI KOGA*, GEOFFREY J. LANE*, ATSUYUKI YAMATAKA* \\ *Department of Pediatric General and Urogenital Surgery, Juntendo University Faculty of Medicine, Tokyo, Japan
}

\begin{abstract}
Robotic surgery is a promising new field in pediatric surgery expected to develop markedly. It involves the application of technology that minimizes stresses related to therapeutic intervention, which will greatly benefit the treatment of children requiring surgery. While it is only a matter of time before robotic surgery is adopted routinely, there are technical issues related to instruments and equipment required to perform the wide range of surgical procedures in patients of various sizes covered by pediatric surgery; in other words, equipment must also be adaptable enough to use from neonates to teenagers. Unfortunately, current robotic instruments are just scaled down adult instruments, for example, instruments used with 2D/3D telescopes scaled down from $8 \mathrm{~mm}$ to $5 \mathrm{~mm}$. We anticipate that, as the number of complex pediatric laparoscopic/thoracoscopic procedures performed with robotic enhancement increases, there will be improvement in the hardware with associated measurable benefits in patient outcomes.
\end{abstract}

Key words: pediatric surgery, robotic surgery, da Vinci system

\section{Introduction}

Developments in laparoscopic surgery have undoubtedly improved postoperative discomfort and morbidity, decreased hospital stay, enhanced recovery, and improved wound cosmesis. The evolution of laparoscopic surgery highlights the transitionary stages that follow the adoption and diffusion of surgical innovation ${ }^{1)}$. There are several limitations to the use of conventional laparoscopic and thoracoscopic surgical techniques such as two-dimensional visualization of the surgical field with loss of depth perception, counterintuitive movements with rigid and non-articulated instruments, loss of eye-hand coordination, and poor ergonomics for the operating surgeon. Robotic surgery was developed to improve these drawbacks. Indeed, robotic surgery offers three dimensional visualization, articulated instruments with seven degrees of freedom, intuitive automation, tremor filtering, motion scaling, complete camera control, and ergonomically ideal positioning for the surgeon. This technology was initially developed for adult surgery but its use has been progressively extended to the field of pediatrics, and the list of applications continues to grow. The lag between what is reported in the literature about robotic surgery lags behind what is in actually being used in clinical practice because of the repaid evolution of technology and its early adoption by eager surgeons resulting in a dearth of good up-to-date reports. Herein we discuss the advantages and limitations of the current technology, and describe the relevant clinical applications of robotics in pediatric surgery.

\section{The da Vinci system}

The two commercially available robotic surgery systems (da Vinci and ZEUS) share a similar design. The da Vinci robotic surgical system (Intuitive Surgical, Sunnyvale, CA, USA) is a computer enhanced telemanipulator currently approved by the U.S. Food and Drug Administra-

Corresponding author: Hiroyuki Koga

Department of Pediatric General and Urogenital Surgery, Juntendo University Faculty of Medicine

2-1-1 Hongo, Bunkyo-ku, Tokyo 113-8421, Japan

TEL: +81-3-3813-3111 FAX: +81-3-5802-2033

E-mail: h-koga@juntendo.ac.jp

334th Triannual Meeting of the Juntendo Medical Society: Future Perspectives of Robotic Surgery in Juntendo〔Held on Sep. 11, 2014〕

[Received Mar. 3, 2015] 
tion (FDA) for adult and pediatric patients. This system comprises three components: a patient-side surgical cart, a vision system, and a surgical console. The surgical cart is composed of three or four arms for controlling a $12 \mathrm{~mm}$ three-dimensional or $5 \mathrm{~mm}$ two-dimensional camera and two or three surgical instruments. The system provides the surgeon with stereoscopic vison, provides motion scaling and tremor reduction, and features instruments capable of 7 degrees of motion, including grip, compared with 4 degrees of motion available with standard laparoscopic instruments. The da Vinci surgical system is the most frequently reported robotic platform used in the pediatric age group at $89 \%$, followed by ZEUS (Computer Motion Inc., CA) at $3.5 \%$, with the remainder of reports failing to stipulate specifically which robotic platform was used $^{1)}$.

\section{Clinical application of robots in pediatric surgery}

The increase in reports about robotic surgery in children has been astounding with over 200 publications appearing in the literature from 52 institutions in 18 countries during 2013. The majority are from North America ${ }^{1}$. The most commonly reported gastrointestinal, genitourinary, and thoracic procedures reported were fundoplication, pyeloplasty, and lobectomy. Essentially, almost all reports described the application of robotic surgery to procedures currently performed using conventional laparoscopy or thoracoscopy. However, there have been several reports published during the past few years describing the techniques for and experience of robot-assistance for procedures in pediatric surgery $^{2)-10)}$.

Initial results of robotic surgery in the field of pediatrics are encouraging with success rates identical to those of conventional laparoscopy. However, questions remain about its role in everyday clinical practice. Is it feasible? Is it safe? Is it efficient? Are there differences between open, laparoscopic, and robotic surgical intervention that will ultimately influence the quality of treatment? Answers to these and other questions will be forthcoming as technology evolves with time, but as a general concept, any procedure that involves complex suturing or dissection can be considered ideal for the da Vinci system. More complex procedures including Kasai portoenterostomy, Mitrofanoff appendicovesicostomy, choledochal cyst excision, and thoracic tumor resection best demonstrate how robotic surgery is an enabling innovation, greatly improving the efficiency of the operator's skill when surgical access is extremely limited or when reconstruction is tedious and complicated. The acquisition of robotic skills is easier and more rapid than mastering laparoscopic skills and less taxing, and laparoscopic skills are not a prerequisite for robotic surgery ${ }^{11)}$.

Instrument size has been the most obvious limiting factor for applying robotic surgery more extensively in children and an issue that has the highest priority. The potential for excellent results in older children and smaller patients weighing less than $5 \mathrm{~kg}$ is good and the expectation is that most procedures currently performed can be accomplished with robotic assistance. A unique feature of surgery in children weighing less than $3 \mathrm{~kg}$ is space. Most procedures currently performed using conventional laparoscopy or thoracoscopy use $3-5 \mathrm{~mm}$ sized instruments, even for neonates. However, robotic instruments have to be longer because of articulations, meaning they protrude more from trocars.

Although the da Vinci platform has been used in neonates weighing as little as $2.2 \mathrm{~kg}$, it is not easy to use in children weighing less than $5 \mathrm{~kg}$. Proper planning and flexibility in determining the bestsuited trocar positions will help overcome these obstacles when compared with standard minimally invasive procedures. Finally, the selection of instrument tips is considerably limited for small patients, particularly for $5 \mathrm{~mm}$ instruments. There are substantial difficulties encountered with conventional endoscopic instruments, including loss of wrist flexibility, poor touch feedback, the fulcrum effect of the body wall while handling instruments, loss of three-dimensional vision, and poor ergonomics ${ }^{12) 13)}$.

Ideally robotic equipment should alleviate these impediments while enhancing motion scaling and stabilizing tremor. In fact, some biomedical engineers believe that laparoscopy is "transitional" technology with robotic surgery as the ultimate form of assisted surgery ${ }^{14)}{ }^{15}$. Cundy et al assessed currently available instruments for robotic surgery 
and found none corresponded to what would work best theoretically with size being a major issue with performance directly affected by size ${ }^{16)}$. Interestingly, the smaller $5 \mathrm{~mm}$ diameter da Vinci instruments have not been well received by pediatric surgeons ${ }^{16)}$. This is owing to a 'snakelike' distal segment design which has a longer articulation axis, rendering it less accommodating in spatially constrained anatomical workspaces that are the norm in infants and young children ${ }^{17)-19)}$. On the other hand, the larger $8 \mathrm{~mm}$ instruments incorporate the compact EndoWrist patented design and many pediatric surgeons will compromise on instrument diameter because of this design feature. A similar scenario exists for the da Vinci laparoscope range. Smaller $5 \mathrm{~mm}$ scopes exist, but they are monoscopic and pediatric surgeons prefer to use larger $8.5 \mathrm{~mm}$ or $12 \mathrm{~mm}$ scopes because they are stereoscopic. The reason for instruments and scopes being larger than necessary is most likely due to technical limitations imposed by physical limitations, i.e., scalability, or resistance to commercialization owing to unsatisfactory current market forecasts.

\section{The future}

With robotic equipment, precision instruments are required, often with computerized components which are designed and serviced by specialist biological engineers. Surgeons can provide valuable advice and input during development but the days of surgeons designing their own instruments are ancient history. Instruments for use in children must also be as small as possible without compromising performance and all kinds of devices are being developed to deal specifically with very small patients. At this stage, a surgeon can only corroborate but once a prototype is developed, clinical testing is necessary. One specific aspect of robotic surgery that has yet to be addressed fully is force feedback.

Currently, surgeons must rely on visual cues to know how much tension and pressure they are applying to tissues, needles, and sutures when operating with surgical robots. Reliable tactile feedback is of greater importance as instruments get smaller because there can be a loss of "feel" as dependence on robotic equipment increases. There are few reports about this and no randomized studies in children in the literature. Published studies are essentially studies with an evidence level of III or level IV according to the Oxford Centre for Evidence-Based Medicine. Further prospective and comparative studies, especially comparisons between robotic and laparoscopic surgery, are necessary to confirm preliminary results. Economic analyses are also indispensable to ensure the viability of robotic technology. In fact, surgical robotic systems are expensive and involve significant operating costs.

\section{Conclusions}

Current robotic surgery is safe and effective for a variety of pediatric surgical procedures, and will continue to find application as technology improves with more extensive applications to more complex procedures enhancing the efficiency of surgery in situations that are less ideal because of size or space limitations. Future developments will focus on technology to allow flexible, smart, robotic instruments with a smaller footprint to be maneuvered into the smaller operative workspaces in children successfully without loss of responsiveness. Robotic surgery has such potential that pediatric surgeons would be wise to get involved early because the future of surgical intervention in children will focus even more on minimally invasive surgery.

\section{References}

1) Cundy TP, Shetty K, Clark J, et al: The first decade of robotic surgery in children. J Pediatr Surg, 2013; 48: 858-865.

2) Alqahtani A, Albassam A, Zamakhshary M, et al: Robot-assisted pediatric surgery: how far can we go? World J Surg, 2010; 34: 975-978.

3) Atug F, Woods M, Burgess SV, Castle EP, Thomas R: Robotic assisted laparoscopic pyeloplasty in children. J Urol, 2005; 174: 1440-1442.

4) Chaer RA, Jacobsen G, Elli F, Harris J, Goldstein A, Horgan S: Robotic-assisted laparoscopic pediatric Heller's cardiomyotomy: initial case report. J Laparoendosc Adv Surg Tech A, 2004; 14: 270-273.

5) Chandra V, Dutta S, Albanese CT: Surgical robotics and image guided therapy in pediatric surgery: emerging and converging minimal access technologies. Semin Pediatr Surg, 2006; 15: 267-275.

6) Goh PM, Lomanto D, So JB: Robotic-assisted laparoscopic cholecystectomy. Surg Endosc, 2002; 16: 216-217.

7) Knight CG, Gidell KM, Lanning D, Lorincz A, Langenburg SE, Klein MD: Laparoscopic Morgagni hernia 
repair in children using robotic instruments. J Laparoendosc Adv Surg Tech A, 2005; 15: 482-486.

8) Lorincz A, Langenburg S, Klein MD: Robotics and the pediatric surgeon. Curr Opin Pediatr, 2003; 15: 262-266.

9) Woo R, Le D, Albanese CT, Kim SS: Robot-assisted laparoscopic resection of a type I choledochal cyst in a child. J Laparoendosc Adv Surg Tech A, 2006; 16: 179183.

10) Zhou HX, Guo YH, Yu XF, et al: Zeus robot-assisted laparoscopic cholecystectomy in comparison with conventional laparoscopic cholecystectomy. Hepatobiliary Pancreat Dis Int, 2006; 5: 115-118.

11) O'Brien ST, Shukla AR: Transition from open to roboticassisted pediatric pyeloplasty: a feasibility and outcome study. J Pediatr Urol, 2012; 8: 276-281.

12) Diodato MD Jr, Damiano RJ Jr: Robotic cardiac surgery: overview. Surg Clin North Am, 2003; 83: 1351-1367, ix.

13) Falk V, Diegler A, Walther T, Autschbach R, Mohr FW: Developments in robotic cardiac surgery. Curr Opin Cardiol, 2000; 15: 378-387.

14) Ballantyne GH, Moll F: The da Vinci telerobotic surgical system: the virtual operative field and telepresence surgery. Surg Clin North Am, 2003; 83: 1293-1304, vii.

15) Satava RM: Emerging technologies for surgery in the 21st century. Arch Surg, 1999; 134: 1197-1202.

16) Cundy TP, Marcus HJ, Hughes-Hallett A, Najmaldin AS, Yang GZ, Darzi A: International attitudes of early adopters to current and future robotic technologies in pediatric surgery. J Pediatr Surg, 2014; 49: 1522-1526.

17) de Lambert G, Fourcade L, Centi J, et al: How to successfully implement a robotic pediatric surgery program: lessons learned after 96 procedures. Surg Endosc, 2013; 27: 2137-2144.

18) Orvieto MA, Large M, Gundeti MS: Robotic paediatric urology. BJU Int, 2012; 110: 2-13.

19) Thakre AA, Bailly Y, Sun LW, Van Meer F, Yeung CK: Is smaller workspace a limitation for robot performance in laparoscopy? J Urol, 2008; 179: 1138-1142; discussion $1142-1143$. 